\title{
Should Low- and Middle-Income Countries Adopt Clinical Guidelines Developed in 'Rich' Countries?
}

\author{
Alan Haycox 1
}

Published online: 7 June 2018

(C) Springer International Publishing AG, part of Springer Nature 2018

I always find conferences where the latest clinical guidelines are presented incredibly informative. The reaction of the audience immediately enables me to distinguish clinicians that practice in rich countries from those that practice in low- and middle-income countries (LMICs). Clinicians from the rich countries listen with rapt attention to ascertain how best this guideline can be incorporated into their clinical practice. In contrast, clinicians from LMICs appear to be far less interested and perhaps even dismissive of the presentation. Such a variation in response emphasises the extent to which clinical guidelines (invariably developed in the context of rich countries) are perceived as having limited relevance to clinicians operating in LMICs. However, such guidelines are strongly promoted in LMICs by pharmaceutical companies, professional organisations, the media and patient advocacy groups, all of which have a vested interest in equating 'newer' with 'better' and in persuading LMICs to adopt guidelines developed by their 'better informed' (i.e. richer) neighbours. As such, strong political, economic and social pressure is placed on LMICs to address complex disease pathways with a clinical guideline 'fix' incorporating the latest drugs and technologies. However, to what extent is it either feasible or desirable for LMICs to automatically adopt guidelines developed by their richer neighbours?

In terms of feasibility, most LMICs perceive their priority as being the delivery of safe, efficient and universal access to 'basic' healthcare rather than promoting the use of expensive patient pathways of care. Therefore, to be of

Alan Haycox

ahay@liv.ac.uk

1 University of Liverpool Management School, Chatham Street, Liverpool L69 7ZH, UK relevance, clinical guidelines must explicitly take account of the limited resources available to many LMICs whether in terms of medical technology or new and expensive drugs. Since 2010, a total of 139 new medicines have been registered by the European Union and many of these 'innovations' were rapidly incorporated into new clinical guidelines. However, out of this total, Bulgaria publicly reimbursed only 44, Croatia reimbursed 27 whilst Serbia reimbursed only 1! [1]. Clearly, for a clinical guideline to be implemented, LMICs require access to the drugs outlined in the pathway.

However, even if the availability of drugs and technology makes it feasible, we must consider whether it is desirable for LMICs to automatically adopt clinical pathways developed in richer countries. The past decade has witnessed a rapid expansion in the development and implementation of clinical guidelines. Such guidelines invariably advocate the use of innovative but frequently expensive drugs; providing funding in a comprehensive and sustainable manner proves difficult for wealthy countries and is normally far beyond the ability or willingness of most LMICs to fund.

A simple but powerful 'benchmark' that LMICs can use when evaluating whether to introduce a clinical guideline is to evaluate the level of 'opportunity cost' that would be imposed as a consequence of its introduction. When considering introducing a new clinical guideline, LMICs should analyse the level and nature of services that would have to be foregone to free up sufficient funds to introduce the guideline. The opportunity costs arising from the introduction of clinical guidelines are likely to be far higher in LMICs than in 'rich' countries where most of the 'low-hanging fruit' (my apologies, I promised myself that I would never use this terrible term; however, unfortunately, in this context it seems appropriate!) in terms of cost- 
effective basic interventions have already been collected. Low- and middle-income countries should therefore only decide to introduce a clinical guideline where the benefits of a guideline introduction exceed the opportunity costs imposed on their health service as a result of the diversion of funds required to introduce such a guideline. In response to proponents of guidelines who argue in favour of their need for clinical 'purity', I would simply point to a range of publications from Alan Williams cogently arguing that it is unethical not to consider the resource implications of clinical decision making. More specifically, the ethical imperative for clinical guidelines to take account of cost effectiveness has been well argued in this very journal [2]. The only situation in which a guideline should be automatically introduced is when it has been proven to be both a better and a cheaper method of supporting patients in any particular therapeutic area. Unfortunately, such guidelines are rare as they usually introduce new and expensive elements into the pathway, which require an assessment of the extent to which the additional benefits are 'worth' the additional costs incurred in introducing the guideline. Such 'trade-offs' have to be made by each individual country in relation to their healthcare priorities, resource availability and the opportunity cost imposed on their health services as a consequence of the introduction of the guideline.

Currently, the decision on whether to introduce a clinical guideline is based on an asymmetrical structure of information. Proponents of the guideline ensure that the benefits to patients arising from the introduction of the guideline are apparent and highly visible. However, the opportunity costs imposed on the health service (as a result of the withdrawal or unavailability of services to other patients as a consequence of a diversion of funds) are neither apparent nor visible. Such asymmetry in the information base risks diverting resources away from areas of national priority such as health promotion (I am continuously surprised at the continuing high rates of smoking in LMICs) or essential vaccination and immunisation programmes. Given such a choice, it appears to be difficult to justify adopting expensive clinical guidelines until more essential interventions providing proven clinical benefits in a cost-effective manner have been fully utilised.

Given the variation in 'affordability' between each individual country, both the countries themselves and European agencies must reject a 'one size fits all' approach to the development and implementation of clinical guidelines in favour of the development of 'bespoke' guidelines tailored to the needs, resource availability and priorities of each individual country. Such guidelines should balance health outcomes, affordability and cost in a manner that ensures that the health resources available to each LMIC are used to optimise the health of the entire patient population served by the bespoke guideline. It is time for agencies charged with supporting healthcare development throughout Europe to switch their focus from the rich countries of Western Europe (whose healthcare systems are already well developed) to the developing healthcare systems in Central, Eastern and Southern Europe. Through the provision of advice and support tailored to the individual needs of each country, such agencies have the opportunity to make a significant contribution to meeting the healthcare needs of the poorer members of the European 'family'. Whilst perhaps unfamiliar when applied in the context of clinical guidelines, such an approach is neither new nor revolutionary. The World Health Organization essential drug initiative emphasises the need to prioritise cost-effective and safe medicines that address priority healthcare needs with due regard to public health relevance.

If LMICs feel the need to 'reach for the stars' by adopting clinical guidelines developed in rich countries, they should do so in the full and certain knowledge that such an approach risks them 'falling back to earth'. They risk placing unnecessary strains on their health services simply to emulate guidelines produced in richer countries. They need to consider very clearly the extent to which the 'stars' are worth such a sacrifice to the populations that they serve.

Acknowledgements This editorial is dedicated to the memory of Alan Maynard, who more than any other person led the development of health economics in the UK.

\section{Compliance with Ethical Standards}

Funding No sources of funding were received for the preparation of this editorial.

Conflict of interest Alan Haycox has no conflicts of interest directly relevant to this content of this editorial.

\section{References}

1. Novakovic T, Martin AP, Parker M, et al. The value of innovation in decision making in health care in Central Eastern Europe: the Sixth International Conference, 2 June 2017, Belgrade, Serbia. Expert Rev Pharmacoecon Outcomes Res. 2017;17(6):519-21. https://doi.org/10.1080/14737.2017.1385394.

2. Boyd K, Hall P. Can clinical guidelines afford to ignore cost effectiveness? An ethical perspective. Pharmacoeconomics. 2016;34(6):529-31. https://doi.org/10.1007/s40273-016-0391-2. 